\title{
FDTD Analysis of Propagation Characteristics of Tera-Hertz Electromagnetic Pulses
}

\author{
Young-Seek Chung, Changyul Cheon, Member, IEEE, Joo-Hiuk Son, Member, IEEE, and \\ Song-Yop Hahn, Senior Member, IEEE
}

\begin{abstract}
A three dimensional finite difference time domain (FDTD) technique is applied to analyze the propagation characteristics of terahertz (THz) antenna whose dimension is smaller than the wavelength. A diffraction theory is also utilized with the FDTD technique for observing the far-field dispersion and attenuation of radiation from the THz antenna with a hyper-hemispherical silicon lens which is much larger than the wavelength. The signal degradation effects due to misalignment between the antenna and silicon lens are also characterized.
\end{abstract}

Index Terms-Diffraction theory, FDTD, terahertz antenna with Si lens.

\section{INTRODUCTION}

$\mathbf{R}$ ECENTLY, terahertz (THz) electromagnetic sources [1], [2] draw much attention and interest because they are essential parts of many potential applications in the sub-millimeter range. The applications include the imaging with terahertz beams [3], far-infrared spectroscopy, millimeter-wave communications, etc. Despite the great importance of terahertz signals, the propagation characteristics of $\mathrm{THz}$ antenna have not yet been studied in detail. However, the exact analysis on the role and characteristics of antenna and silicon ( $\mathrm{Si}$ ) lens in the $\mathrm{THz}$ system becomes more important as we want to get stronger signal and wider spectrum.

Some field analyzes on the $\mathrm{THz}$ antenna have been performed using geometrical optics and diffraction theory [4]. However, these analytic aproaches have limitations in modeling the excitation current driving the antenna and the complicated antenna structures with bias metal lines. Therefore, full wave analysis such as finite difference time domain (FDTD) technique is required to study the attenuation and dispersion characteristics in the time domain. But the full wave analysis is not suitable for the analysis of $\mathrm{THz}$ antenna with a large Si lens which helps collimate the radiation because the size of Si lens is several ten times lager than the wavelength. The analytical method such as

Manuscript received October 25, 1999. This paper work was supported by the Korean Ministry of Science and Technology through Creative Research Initiative Program, Seoul, Korea.

Y.-S. Chung and S.-Y. Hahn are with the School of Electrical Engineering, Seoul National University, Shillim-dong, Kwanak-gu, Seoul 151-742, Korea (e-mail: yschung@eesri-10.snu.ac.kr; syhahn@plaza.snu.ac.kr).

C. Cheon is with the Electrical Engineering Department, University of Seoul, 90 Cheonnong-dong, Tongdaemun-gu, Seoul 130-743, Korea (e-mail: changyul@uoscc.uos.ac.kr).

J.-H. Son is with the Department of Physics, University of Seoul, 90 Cheonnong-dong, Tongdaemun-gu, Seoul 130-743, Korea (e-mail joohiuk@uoscc.uos.ac.kr).

Publisher Item Identifier S 0018-9464(00)05647-8.

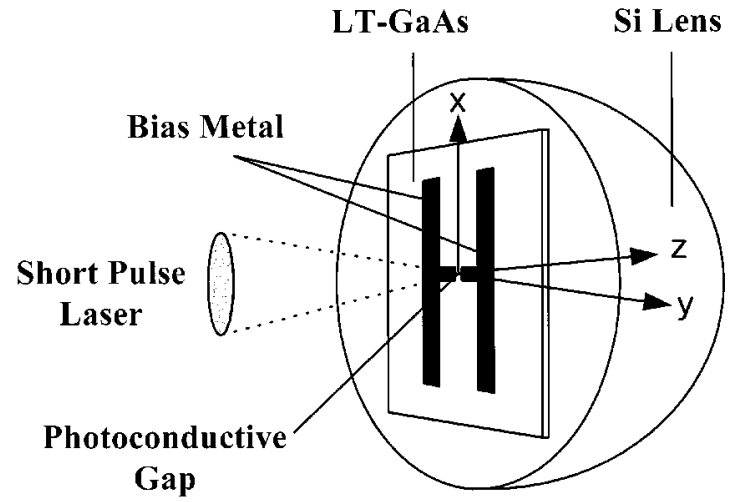

Fig. 1. THz dipole antenna with a hyper-hemisperical Si lens.

diffraction theory also needs to be adopted for analyzing a propagation system with large elements, Si lens in this case.

In this paper, we also suggest a hybrid method combining the FDTD technique and diffraction theory in the time domain for modeling the $\mathrm{THz}$ antenna with a hyper-hemispherical Si lens to study the radiation charateristics of terhertz signals.

\section{TERAHERTZ ANTENNA}

One of the techniques generating $\mathrm{THz}$ electromagnetic waves is to use a microstrip line antenna fabricated on a semiconductor substrate with a dc bias between the lines. And the gap between the lines is driven by a femtosecond laser to give transient photocurrent which results in the THz radiation. Fig. 1 shows a $\mathrm{THz}$ dipole antenna structure with a hyper-hemispherical $\mathrm{Si}$ lens. A dc voltage is biased at the photoconductive gap through the metal lines.

When a femtosecond laser illuminates the photoconductive gap, electron-hole pairs are generated and they recombine in a few hundred femtoseconds (fs). These photogenerated carriers induce the electric current in the photoconductive gap and the time-vaying current radiates terahertz electromagnetic wave into the free space. To obtain wide spectrum of $\mathrm{THz}$ signal, we use low-temperature-grown GaAs (LT-GaAs) for the semiconductor substrate. The carrier lifetime of LT-GaAs is shorter than $600 \mathrm{fs}$. To enhance the signal strength, a hyper-hemispherical Si lens is attached directly to the back of the antenna substrate. This hyper-hemispherical Si lens increases the directivity of $\mathrm{THz}$ radiation. To detect the $\mathrm{THz}$ electromagnetic waves, the same dipole antenna structure is used. 


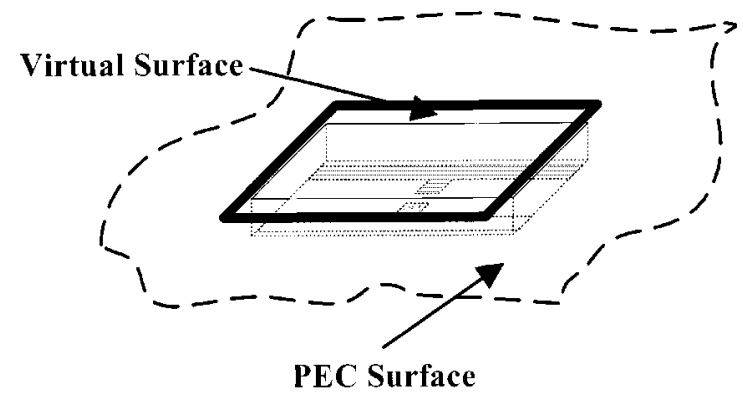

Fig. 2. A virtual surface to calculate the far fields.

\section{FDTD ALGORITHM}

The time and space dependent Maxwell's equations are discretized in both time and space using the FDTD technique that was introduced by Yee [6]. This method needs not vector or scalar potentials. Instead, FDTD is based on the electric and magnetic fields in time and space. Equation (1) represents the finite difference relations for the $x$ component of electric and magnetic field in three dimensional space.

The photocurrents in the photoconductive gap are regarded as the wave sources or the excitation currents. Hence, the photocurrents are added to $y$ component electric fields which are located at the photoconductive gap.

To truncate the computational domain and reduce the reflection at the outer boundaries, absorbing boundary condition $(\mathrm{ABC})$ is required. In this paper, the 2 nd order dispersive boundary condition (DBC) [7] is employed, that is one of $A B C$ 's that show good absorbing performances over a wide frequency band.

\section{FAR FIELD CALCULATION}

To obtain the far fields of the $\mathrm{THz}$ antenna, the virtual surfaces have to be placed in the computational domain and to enclose the whole antenna, where the equivalent electric and magnetic currents are defined. In this paper, we assume that the $\mathrm{THz}$ dipole antenna radiates the electromagnetic wave in the vicinity of the photoconductive gap and the radiation far from the gap can be neglected. Therefore, the virtual surface is assumed to be an aperture surrounded by PEC's which is placed at the upper location of GaAs substrate as Fig. 2.

So, we can assume that the electromagnetic wave is radiated only from the virtual surface.

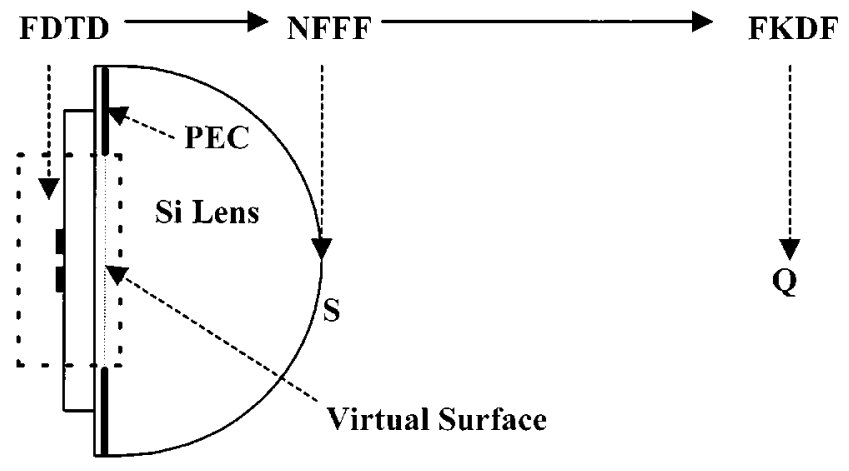

Fig. 3. Far field calculation scheme when Si lens is attached.

If there is no Si lens, the far fields in free space can be obtained by near-field to far-field (NFFF) transformation using the equivalent sources on the virtual surface as

$$
\begin{aligned}
\vec{E}(\vec{r}, t)= & \frac{\mu_{0}}{4 \pi r} \oint_{S}\left\{\hat{r} \times \hat{r} \times \frac{\partial}{\partial t^{\prime}} \vec{J}_{s}\left(\vec{r}^{\prime}, t^{\prime}\right)\right. \\
& \left.+\frac{1}{\eta_{0}} \hat{r} \times \vec{M}_{s}\left(\vec{r}^{\prime}, t^{\prime}\right)\right\} d s^{\prime}
\end{aligned}
$$

where $S$ is a virtual surface and the retarded time is $t^{\prime}=t-(r-$ $\left.\hat{r} \cdot \vec{r}^{\prime} / c\right) \geq 0$. And $c$ is the light velocity in free space. $\vec{J}_{s}$ is an equivalent electric current source and $\vec{M}_{s}$ is a magnetic current source.

When a truncated Si lens is attached as in Fig. 3, we assume that the right half plane is filled with $\mathrm{Si}$ and the virtual surface surrounded by PEC locates in a homogeneous Si material. And we assume that the effects of the reflective waves at the surface of Si lens can be neglected. Because the size of Si lens is very large compared with the wavelength, the interference by the reflected wave can be neglected. So, the far fields can be calculated by two stages as Fig. 3 .

First, using the NFFF transformation and the equivalent sources in the virtual surface, the electric and magnetic fields on the surface of Si lens can be obtained. Second, using Fresnel-Kirchhoff's diffraction formula (FKDF) which is used in optics [8], we can calculate the far fields in free space. Equation (3) is time domain Fresnel-Kirchhoff's diffraction integral.

$$
\begin{aligned}
\Psi_{Q}= & \frac{-1}{4 \pi c} \frac{\partial}{\partial t} \int_{S} \frac{\Psi_{S}(t-r / c)}{r} \\
& \cdot\left(\cos \theta_{\text {out }}-n_{i} \cos \theta_{\text {in }}\right) d s^{\prime}
\end{aligned}
$$

$$
\begin{aligned}
& \left.E_{x}\right|_{i, j, k} ^{n+1}=\left.E_{x}\right|_{i, j, k} ^{n}+\frac{\Delta t}{\varepsilon_{i, j, k}}\left(\frac{\left.H_{z}\right|_{i, j+1 / 2, k} ^{n+1 / 2}-\left.H_{z}\right|_{i, j-1 / 2, k} ^{n+1 / 2}}{\Delta y}-\frac{\left.H_{y}\right|_{i, j, k+1 / 2} ^{n+1 / 2}-\left.H_{y}\right|_{i, j, k-1 / 2} ^{n+1 / 2}}{\Delta z}\right) \\
& \left.H_{x}\right|_{i, j, k} ^{n+1}=\left.H_{x}\right|_{i, j, k} ^{n-1 / 2}+\frac{\Delta t}{\mu_{i, j, k}}\left(\frac{\left.E_{y}\right|_{i, j, k+1 / 2} ^{n}-\left.E_{y}\right|_{i, j, k-1 / 2} ^{n}}{\Delta z}-\frac{\left.E_{z}\right|_{i, j+1 / 2, k} ^{n}-\left.E_{z}\right|_{i, j-1 / 2, k} ^{n}}{\Delta y}\right)
\end{aligned}
$$




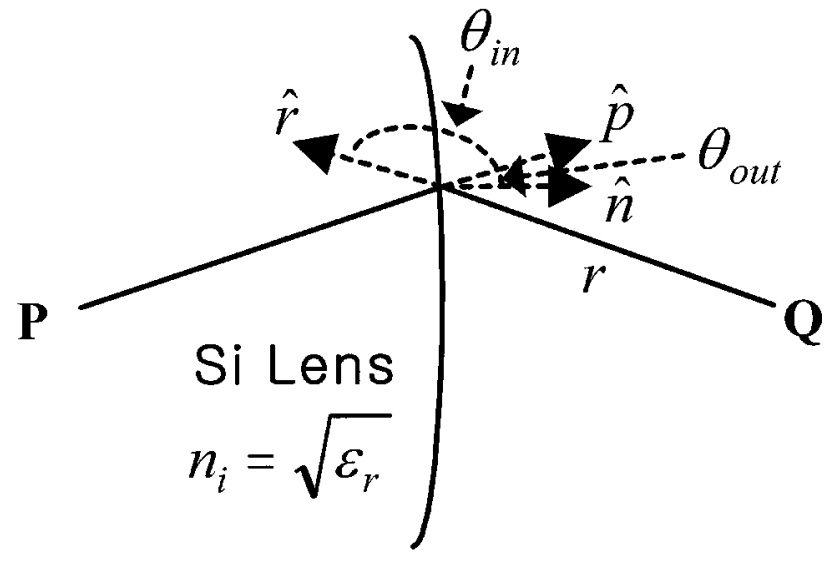

Fig. 4. Fresnel-Kirchhoff's scheme in detail.

TABLE I

SPECIFICATIONS OF THZ DIPOLE ANTENNA

\begin{tabular}{lc}
\hline \hline Thickness of GaAs $(\mu \mathrm{m})$ & 500 \\
\hline Dipole width/length $(\mu \mathrm{m})$ & $10 / 40$ \\
\hline Permittivity $\left(\varepsilon_{\mathrm{r}}\right)$ & 13.2 \\
\hline Rising/Falling time $(\mathrm{ps})$ & $0.2 / 0.4,1.0,2.0$ \\
\hline Size of the virtual surface $(\mu \mathrm{m})$ & $210 \times 125$ \\
\hline \hline
\end{tabular}

where $S$ is the surface area of Si lens, $\Psi_{S}$ is the scalar fields on the surface of Si lens $r$ is the distance between the surface of $\mathrm{Si}$ lens and the far point $Q$. And $n_{i}$ is the refractive index of $\mathrm{Si}$.

Fig. 4 represents Fresnel-Kirchhoff's diffraction scheme in the vicinity of the surface of Si lens in detail.

where $\hat{n}$ is a normal vector, $\hat{r}$ is a vector from the far point $Q$ and $\hat{p}$ is a vector from the center of the virtual surface.

\section{NUMERICAL RESULTS}

\section{A. THz Antenna without Si Lens}

Considering the symmetric relation of the $\mathrm{THz}$ dipole antenna structure and the excitation currents, we can place the PEC and PMC planes at the boundary surfaces so that the computational domain is reduced by a factor of 4 . The reduction of computational domain makes it possible to decrease the computational time and the usage of computer memory. The specifications of $\mathrm{THz}$ dipole antenna are given in Table I. GaAs substrate is assumed to be lossless and not to be dispersive. And the thickness of the metal lines is neglected. The bias metal lines are assumed to be infinite along the $y$ axis.

Fig. 5 shows the reduced THz dipole antenna model considering the symmetric antenna structure and the boundary conditions such as PEC or PMC. The excitation currents are assumed to flow only in the photoconductive gap on the surface of GaAs substrate.

Fig. 6 represents the electric fields at some points on the virtual surface. As the distance increases from the center of the virtual surface, the magnitude of the electric field decreases. From the result of Fig. 6, we can see that the assumption that

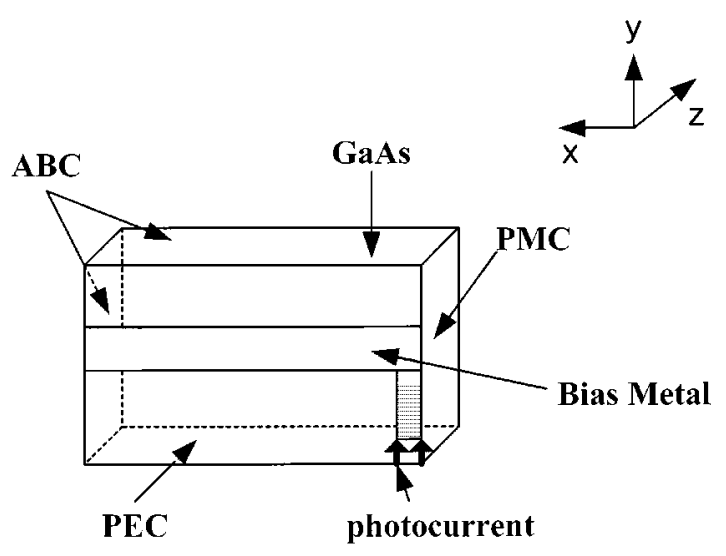

Fig. 5. The reduced $\mathrm{THz}$ dipole antenna model.

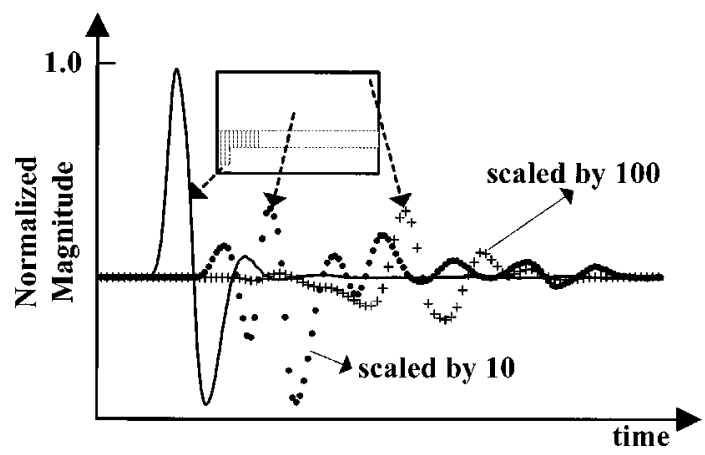

Fig. 6. Field waveforms on the virtual surface. Solid line represents the field at the center of the photoconductive gap, circle represents the field in the middle of the virtual surface and ' + ' represents the field at the corner.

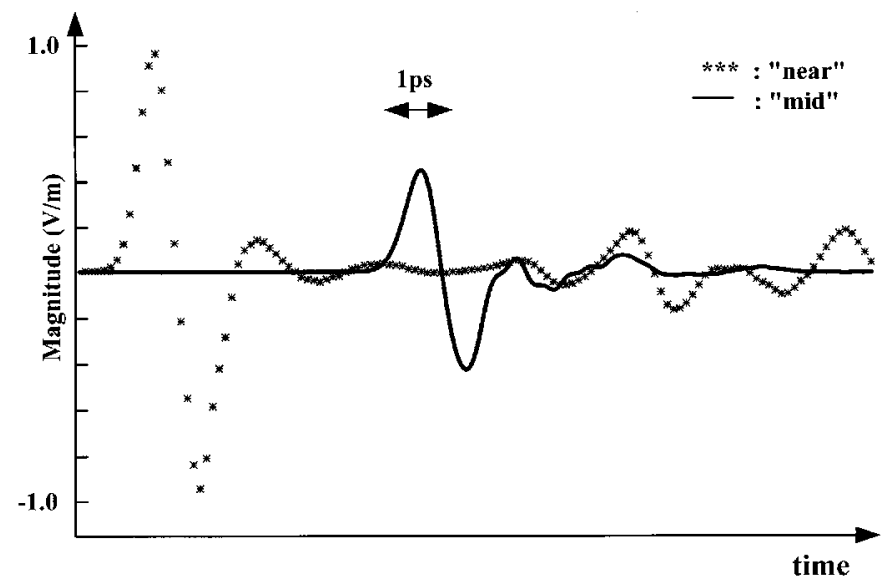

Fig. 7. Electric field waveforms at "near" and "mid" point. The "near" field is normalized. The magnitude of the "mid" field is relative to that of the "near" field.

the electromagnetic wave is radiated only from the virtual surface is reasonable. Fig. 7 represents that the time domain electric field waveforms at "near" and "mid" point along the $z$ axis are displayed when the source current has $0.2 \mathrm{ps}$ rising and 0.4 ps falling time. The label "near" denotes the location in the middle of GaAs substrate and "mid" is at the virtual surface. In the Fig. 7, the "near" field has a ringing tail. We expect that this ringing is due to the multi-reflection in GaAs substrate. The "mid" field has a small ringing tail. 


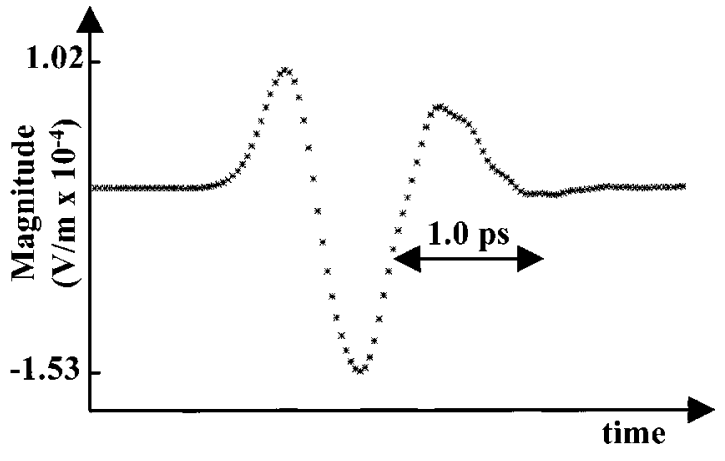

Fig. 8. Far field waveform at the $z$ axis in time domain.

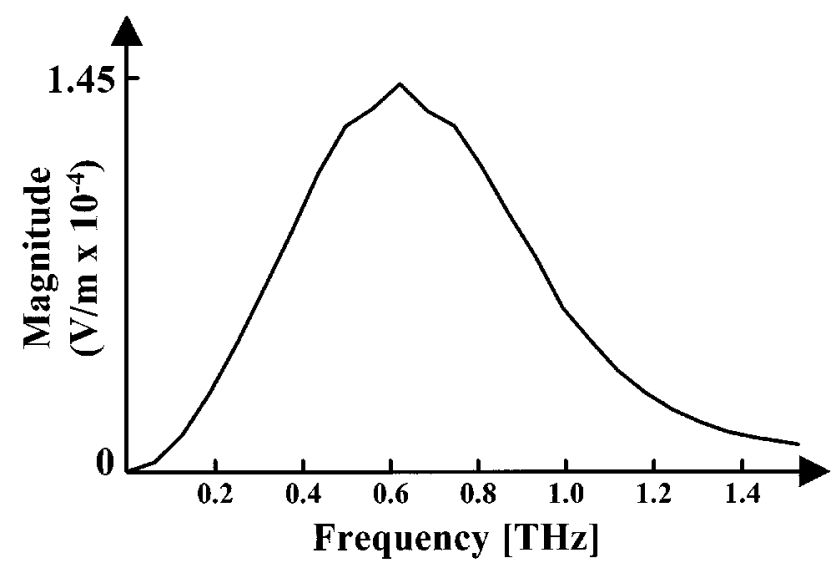

Fig. 9. Far field waveform at the $z$ axis in frequency domain.

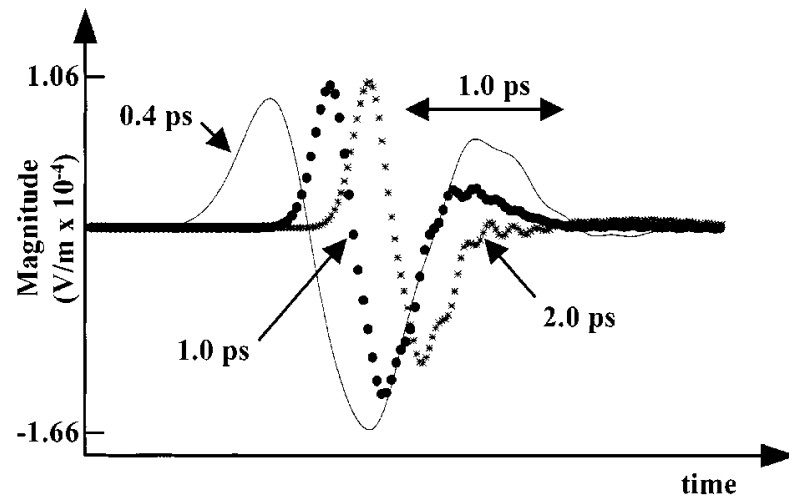

Fig. 10. Far field waveforms for different excitations. The 2nd peak decreases as the falling time increases.

Figs. 8 and 9 represent the far field waveform at the $z$ axis in time and frequency domain respectively. The magnitude of the far field is relative to that of the "near" field. The radiated field has a wide spectrum with the strongest field at around $0.6 \mathrm{THz}$.

Fig. 10 shows the far field waveforms with the various falling times of the excitation current. Larger the falling time, lower the 2nd peak value as expected.

\section{B. THz Antenna with a Hyper-Hemispherical Si Lens}

The specifications of Si lens are shown in Table II. The photocurrent is assumed to have $0.2 \mathrm{ps}$ rising time and $0.4 \mathrm{ps}$ falling time in this case.
TABLE II

SPECIFICATIONS OF HYPER-HEMISPHERICAL SI LENS

\begin{tabular}{ll}
\hline \hline Radius $(\mathrm{mm})$ & 5.0 \\
\hline Flat $(\mathrm{mm})$ & 1.41 \\
\hline Permittivity $\left(\varepsilon_{\mathrm{r}}\right)$ & 11.0 \\
\hline \hline
\end{tabular}

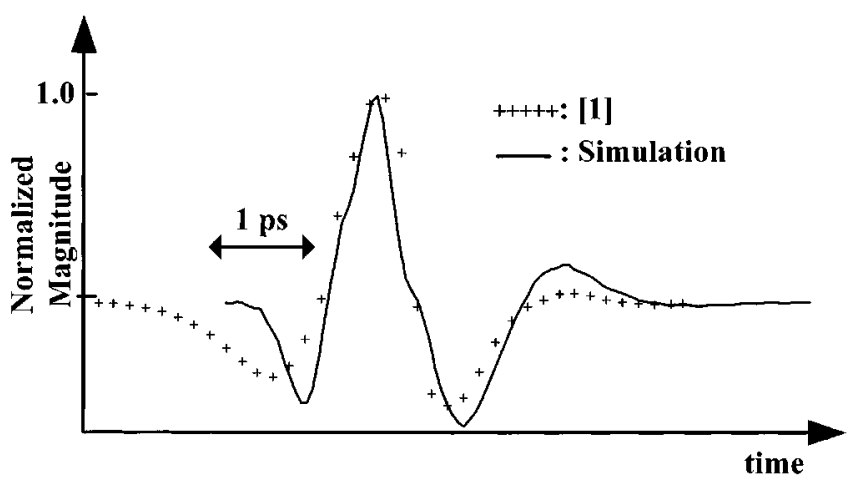

Fig. 11. Comparison with the experimental result of [1].

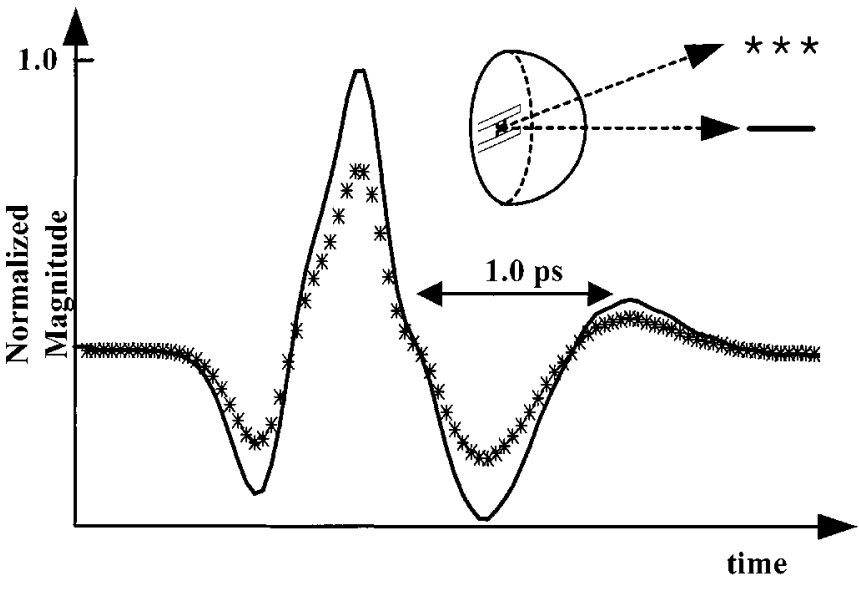

Fig. 12. Far field waveform comparison between at on- and off-axis. The solid line is the case of on-axis and '*' is of off-axis.

Fig. 11 represents the comparison of the normalized time domain far field at the $z$ axis between our simulation and the experimental result of [1].

Fig. 12 shows the electric field waveforms on- and off-axis. On-axis means the position locates at the $z$ axis and off-axis means the position locates E-plane at an angle of 15 degree from the $z$ axis.

Fig. 13 shows the normalized magnitude in frequency domain effects of the misalignment between of $\mathrm{THz}$ dipole antenna and Si lens. If there is a misalignment, the higher frequency term decreases. We think that these phenomena may be mainly due to the increase of the reflection loss at the surface of Si lens if misaligned.

\section{CONCLUSION}

The propagation characteristics of the dipole antenna with $\mathrm{Si}$ lens radiating $\mathrm{THz}$ electromagnetic waves are analyzed using 


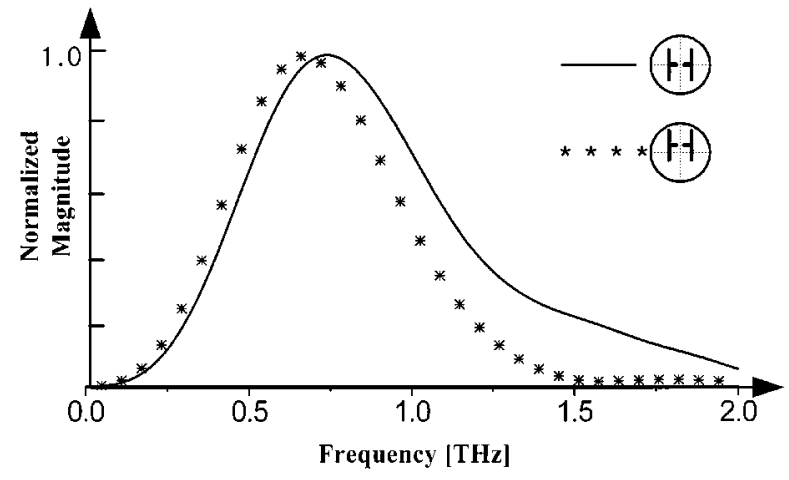

Fig. 13. Antenna misalignment effect in frequency domain. The solid line is the case of the exact alignment and ' $*$ ' is of the misalignment with $0.5 \mathrm{~mm}$ shift to the $x$ axis.

the FDTD technique and Fresnel-Kirchhoff's diffraction formula. The electric and magnetic fields near the antenna are obtained and saved using FDTD technique. Without Si lens, the far field waveforms are calculated using NFFF transformation only. When Si lens is attached, the far field signals are obtained using NFFF transformation and Frensel-Kirchhoff's diffraction integral.

\section{REFERENCES}

[1] D. Grischkowsky, S. Keiding, M. van Exter, and Ch. Fattinger, "Farinfrared time-domain spectroscopy with terahertz beams of dielectrics and semiconductors," J. Opt. Soc. Am. B, vol. 7, p. 2006, 1990.

[2] J.-H. Son, T. B. Norris, and J. F. Whitacker, "Terahertz electromagnetic pulses as probes for transient velocity overshoot in GaAs and $\mathrm{Si}$," J. Opt. Soc. Am B, vol. 11, p. 2519, 1994.

[3] B. B. Hu and M. C. Nuss, "Opt. Lett.,", vol. 20, p. 1716, 1995.

[4] P. U. Jepsen and S. R. Keiding, "Radiation patterns from lens-coupled terahertz antennas," Opt. Lett., vol. 20, pp. 807-809, 1995.

[5] M. van Exter and D. Grischkowsky, "Phys. Rev.,", vol. 41, p. 12140, 1990.

[6] K. S. Yee, "Numerical solution of initial boundary value problems involving Maxwell's equations in isotropic media," IEEE Trans. and Antennas and Propagation, vol. 14, pp. 302-307, 1996.

[7] Z. Bi, K. Wu, C. Wu, and J. Litva, "A dispersive boundary condition for microstrip component analysis using the FD-TD method," IEEE Trans. MTT, vol. 40, pp. 774-777, 1992.

[8] M. Born and E. Wolf, Principles of Optics: Cambridge Univ. Press, 1980.

[9] A. Taflove, Computational Electrodynamics: The Finite Difference Time Domain Method. Boston, London: Artech House, 1995.

[10] - Advances in Computational Electrodynamics: The Finite-Difference Time-Domain Method. Boston, London: Artech House, 1998.

[11] C. A. Balanis, Antenna Theory Analysis and Design. New York, Singapore: John Wiley \& Sons, 1982. 